\title{
Serum Interleukin-33 in Rheumatoid Arthritis and its Relation to Disease Activity
}

\author{
Mai S. Shalaby', Mona I. Salama², Rasha E. Badr², Amany M. Hassan', \\ Amal S. Ahmed', and Aziza S. Omar ${ }^{3}$
}

${ }^{1}$ Clinical Pathology, Dept., Faculty of Medicine, Suez Canal University; ${ }^{2}$ Clinical Pathology Dept., Faculty of Medicine, Port Said University; ${ }^{3}$ Rheumatology and Rehabilitation Dept., Faculty of Medicine, Suez Canal University, Egypt

\begin{abstract}
Background: Rheumatoid Arthritis (RA) is a chronic progressive, systemic inflammatory, connective tissue disease affecting approximately $1 \%$ of the general population. Interleukin-33 (IL33), a member of the IL-1 family, is a ligand for the orphan receptor ST2 (known as IL-1RL1 also). IL-33 is crucial for Th2 cytokine-mediated immune responses however; it can overcome this role in RA. Aim: This study aimed to investigate the potential role of inflammatory cytokine (IL-33) in RA and assess the correlation of IL-33 level to disease activity. Subjects and Method: The study included sixty patients with RA. Patients were diagnosed according to the American College of Rheumatology (ACR) 2010 revised criteria and were classified into 2 groups of 30 patients each according to disease activity score 28 (DAS28), the first group included RA patients with DAS28 of $\leq 2.4$ and the second group included RA patients with DAS28 $>2.4$.Thirty normal subjects served as a control group. Serum IL-33 was measured using ELISA. Results: Serum IL33 level was significantly higher in patient's group compared to the control group $(\mathrm{P} \leq .001)$. Also, serum IL33 level, was significantly higher in patients' group 2 (DAS>2.4) than patients' group 1 (DAS $\leq 2.4$ ) $(P<0.001)$. Serum IL-33 level positively correlated with disease duration, ESR, CRP and disease severity. Conclusion: IL-33 is a novel potential marker for the risk of RA as well as a marker of disease activity
\end{abstract}

Key words: Rheumatoid arthritis, cytokine, IL-33, disease activity

\section{Introduction}

RA is an autoimmune disease characterized by chronic inflammatory response, including synovial proliferation and excessive proinflammatory cytokine production, leading to eventual cartilage and bone destruction. Several proinflammatory cytokines are considered critical in forming the inflammatory process of RA, including IL-1, IL-6, IL-8, IL-15, and tumor necrosis factor-alpha $(\text { TNF- } \alpha)^{(1)}$. IL-33 is a novel member of IL-1 family. At the protein level, IL-33 is broadly expressed in multiple tissues and organs especially enriched in the central nervous system and gastrointestinal $\operatorname{tract}^{(2)}$. IL-33had been implicated in several inflammatory and autoimmune diseases. IL-33 can be produced by various types of tissues and cells 
and induce gene expression of $\mathrm{T}$ helper 2 (Th2)-associated cytokines via binding to the orphan receptor ST2. By promoting Th2 type immune response, IL-33 plays important roles in the allergy, whereas its function in autoimmune diseases attracts more attention(3). There has been much evidence confirming the involvement of IL-33 in rheumatoid arthritis. In earlier studies, it was reported that administration of $\mathrm{SST} 2$ fusion protein dramatically attenuated disease severity, which contains reducing cellular infiltration in the joints, synovial hyperplasia, and joint erosion, by inhibiting the release of proinflammatory cytokines comprising IL-6, IL12, TNF-alpha, and interferon- gamma $(\text { IFN- } \gamma)^{(4)}$. After that, the high expression levels of IL-33 in human RA synovium and experimental arthritis were discovered. Moreover, treatment with an ST2 blocking antibody at the onset of disease at tenuated the severity of collagen induced arthritis ( $\mathrm{CIA})$ and reduced joint destruction, which totally suggested that locally produced IL-33 may contribute to the pathogenesis of joint inflammation and destruction ${ }^{(5)}$. Many studies reported the correlation of IL-33 with rheumatic diseases, and most of them found that IL-33 expression levels were consistent with disease activity and development. Furthermore, evidence has indicated that IL33-related treatment may ameliorate the pathogenic conditions and attenuate disease progression of those rheumatic diseases. Therefore, elucidation of the roles of IL-33 in rheumatic diseases would be beneficial to understand the pathogenesis and therapy of these diseases(3). IL-33, which has been demonstrated to induce cytokine synthesis and mediate inflammatory responses through its receptor ST2 and it is widely expressed in many tissues such as the liver, lung, central nervous system, and multiple types of cells including epithelial cells, endothelial cells, smooth muscle cells, macrophages, and fibroblasts $^{(6)}$. IL-33 is expressed in the synovium of patients with RA, and IL-33 serum concentrations are elevated in patients with RA. These findings suggest that IL-33 is involved in RA pathogenesis. This concept is supported by data obtained in collagen induced arthritis (CIA). Treatment with a monoclonal antibody against $\mathrm{T}_{1} / \mathrm{ST}_{2}$ or genetic ablation of T1/ST2 ameliorated arthritis ${ }^{(5)}$. Accordingly, the abnormally elevated level of IL-33 in RA patients was correlated with disease activity compared to the moderate or low activity group or healthy volunteers, and for synovial fluid, IL-33 levels were higher than those in sera. These observations revealed that IL-33 was mainly produced in inflamed joints(7).In patients with rheumatoid arthritis; the level of SST2 is elevated in the serum. However, little is known about the levels of IL-33 in rheumatoid arthritis patients ${ }^{(8)}$. In this study, we measure the level of IL-33 in sera of RA patients and examine whether this level could reflect the disease activity.

\section{Subject and Methods}

A cross sectional study was carried out at SCU, FOM teaching hospital. Patients were recruited from the outpatient clinic of the Rheumatology and Rehabilitation department. Lab work took place at clinical pathology department. Study population Include 60 RA patients and 30 healthy controls. The patients were divided into two groups using DAS28 score (A scoring system for RA that depends on a complex calculation based on several tender and swollen joints and ESR). Group I included thirty RA patients with good and moderate activity (DAS28 of $\leq 2.4$ )as defined by 2010 revised American College of Rheumatology criteria (ACR). While group II includes thirty RA patients with no activity (DAS28 > 2.4), Group III includ- 
ed 30 normal, healthy age and gender matched blood donors with no history of autoimmune or rheumatic disease or DMARDS. A written informed consent was obtained from all participants before starting. All patients were subjected to interview, with full history: age, gender, age at diagnosis, duration of disease and treatment taken. Physical examination routine, laboratory measurements [including complete blood counts ( $C B C)$, serum $C$ reactive protein ( $C R P)$ and erythrocyte sedimentation rate (ESR)], Assessment of disease activity status using DAS 28 at the time of blood sampling.

Assessment of serum IL-33.

This was done using Enzyme-linked Immunosorbent assay (ELISA) (Merck KGaA, Darmstadt, Germany) according to the manufacturer's instructions. Procedure: All reagents, working standards, and samples were prepared according to the manufacturer's instructions. $100 \mu \mathrm{l}$ of Assay Diluent was added to each well, then $100 \mu \mathrm{l}$ of Standard, control, or sample were added to each corresponding well and covered with the adhesive strip provided. Plate was incubated for 2 hours at room temperature on a horizontal orbital microplate shaker set at $500 \mathrm{rpm} \pm 50$ $\mathrm{rpm}$. A plate layout was delivered to document standards and samples assayed. Contents were aspirated, then each well was washed with Wash Buffer $(400 \mu \mathrm{l})$, the process repeated three times for a total of four washes. After the last wash, any remaining was removed by wash Buffer by aspirating or decanting. $200 \mu$ of Human IL-33 Conjugate were added to each well and covered with a new adhesive strip for 2 hours at room temperature on the shaker. The aspiration/wash in step 4 was repeated. $200 \mu \mathrm{l}$ of Substrate Solution were added to each well and incubated away from light for 30 minutes at room temperature. $50 \mu$ of Stop Solution were added to each well. The optical density of each well determined within 30 minutes by microplate reader.

Calculation of results: Standard curve created by plotting the mean absorbance for each standard on the $y$-axis against the concentration on the $x$-axis and were drawn a curve on a log/log

\section{Results}

Study population characteristics: The mean age for the control group is 35.47 year compared to 44.29 year in group 2 and 41.1 year in group 1, RA patients in group 1 included 28 (93.33\%) females and $2(6.67 \%)$ males; the patients in group 2 included 24 (80\%) females and 6 (20\%) males. A statistically significant difference was detected between RA patients in group 1 and 2 regarding sex with $P$ value $<0.05 . C R P, E S R$, and IL-33 levels were higher in-patient groups than control group. It was also higher in group 2 than in group 1. The mean CRP level in group1 was 5.1 , and it was 58.47in group 2. A highly significant higher CRP level were detected in group 2 when compared to group $1(P=0.38)$. Also, CRP is significantly higher in both patients' group than the control group. CRP levels were significantly higher in group 2 when compared with group1 and control $(\mathrm{P}=<.005)$. The mean ESR level in the study group1 was (29.4), in the study group 2 was (88.7) and in the control group was (13.07). A statistically significant difference was detected when comparing both study groups (group 1 and group 2) with control $\mathrm{P} \leq$.001and respectively, also statistically significant difference between group1 and 2 was also detected $(P \leq .001)$

IL-33 serum level RA activity: The IL33 serum level in group 1 ranged from 50-65 with (57.97 \pm 20.19$)$. While the IL33 level in group 2 ranged from $170-400$ with $(277.4 \pm 120.2)$. 
IL-33 serum level in RA patient's vs. Control

IL-33 showed statistically significant higher level in both study groups when compared to the control group ( $\left.1, p_{2}=\leq .001\right)$ and showed statistically significant higher levels in group 2 when compared to group 1 (p3=s.001) (Figure 1). Table 1 shows disease duration in both patient groups. In group 1, mean disease duration \pm SD was $7.77 \pm 4.19$ years. Group 2 mean disease \pm SD duration22.76 \pm 8.71 . A statistically significant difference was present between both groups $(p=\leq .001)$. As shown in Figure
(2), a positive correlation between disease duration and IL-33 in patient groups was found. Figure 3 shows a positive correlation between ESR and IL-33 in patient groups. while Figure 4 shows a positive correlation between CRP and IL-33 in patient groups. Table 2 revealed strong positive correlations between CRP, and each of ESR, IL-33, and disease duration and between ESR and each of IL-33 and disease duration and finally a strong positive correlation between IL-33 and disease duration.

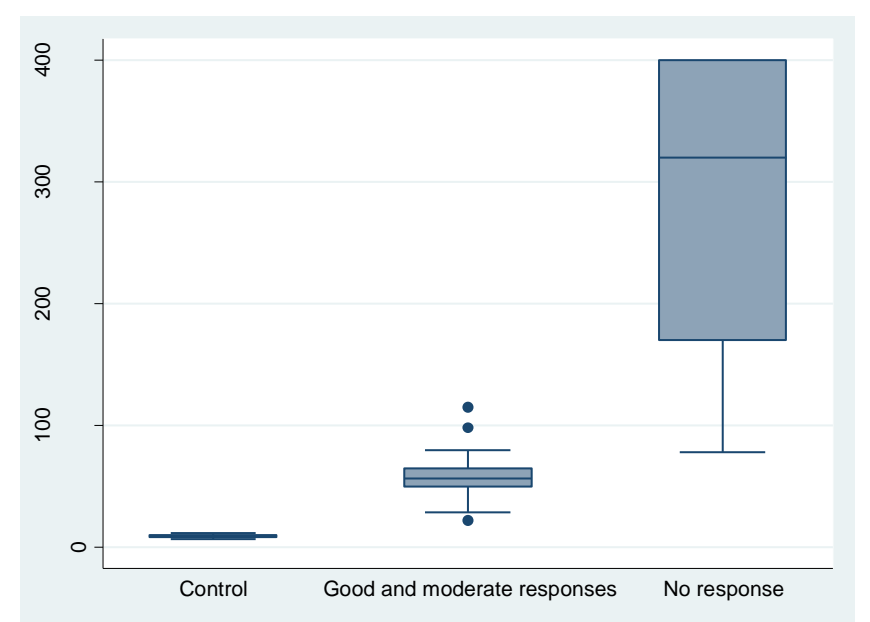

Figure 1: Box plot showing IL-33 levels among study groups

Table 1: comparison of study groups according to disease duration

\begin{tabular}{|l|c|c|c|c|}
\hline $\begin{array}{l}\text { Disease } \\
\text { Duration }\end{array}$ & Group1 $(\mathrm{n}=30)$ & Group2 $(\mathrm{n}=30)$ & Control $(\mathrm{n}=30)$ & $\begin{array}{c}\text { Test of sig. } \\
\mathrm{p} \text {-value }\end{array}$ \\
\hline Mean \pm SD & $7.77 \pm 4.19$ & $22.77 \pm 8.71$ & - & $\mathrm{t}=-8.4978$ \\
\cline { 1 - 3 } Median & 7.5 & 23 & - & \multirow{2}{*}{$-0.0001 *$} \\
\hline Range & $1-18$ & $7-38$ & - & \\
\hline
\end{tabular}

\section{Discussion}

RA is a common connective tissue disorder affecting approximately $1 \%$ of the population(9). RA is a chronic, progressive, systemic inflammatory state of indefinite cause. It is typified by synovial proliferation and a symmetric erosive arthritis of peripheral joints. The hallmark feature of the disease is persistent symmetric poly- arthritis (synovitis) that affects the hands, wrists and feet, although almost all diarthrodial joints may become involved(10). RA is one of the most frequent causes of physical disability among adults with a serious economic burden ${ }^{(11)}$. Different prevalence of the disease throughout the world indicate that genetic factors play an important role in RA pathogenesis ${ }^{(12)}$. The pathogenesis of RA is a complex process 
mediated by a codependent interaction of cytokines, prostanoids and proteolytic enzymes. The levels of representative proinflammatory cytokines are increased in patients with RA compared with other forms of arthritis ${ }^{(13)}$. Many cytokines are

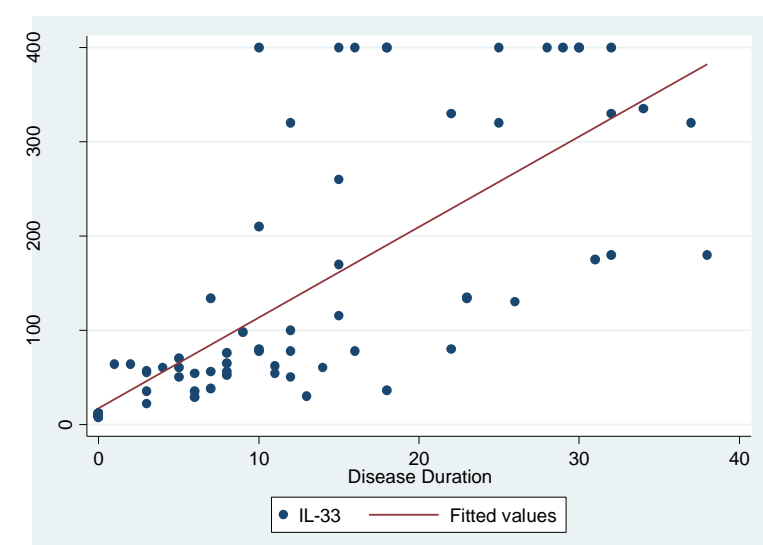

Figure 2: Scatterplot showing correlation between IL-33 and disease duration active in the joints of patients with RA. It is now clear that these cytokines play a fundamental role in the processes that cause inflammation, articular destruction and the co-morbidities associated with $\mathrm{RA}^{(14)}$.

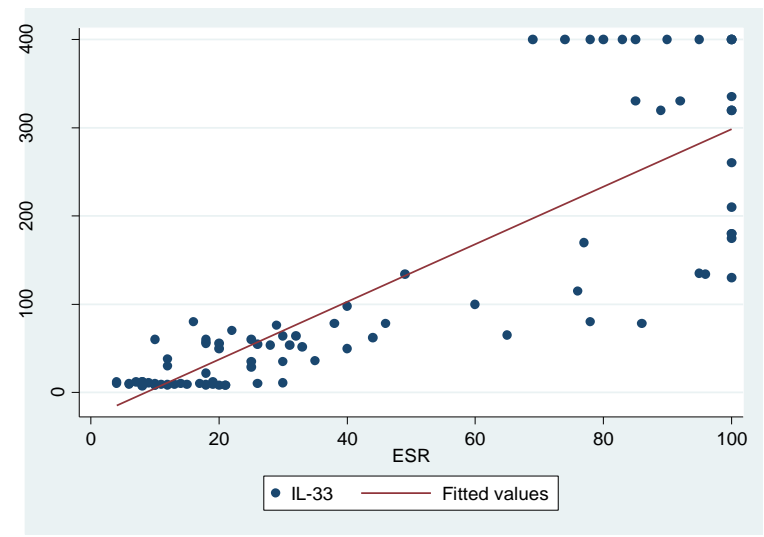

Figure (3) Scatterplot showing correlation between IL-33 and ESR

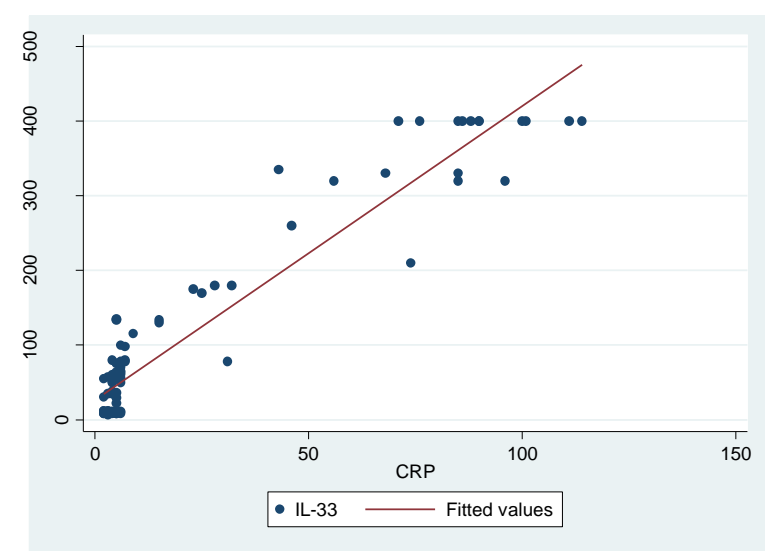

Figure 4: Scatter plot showing correlation between IL-33 and CRP

Table 2: Spearman correlation between laboratory results.

\begin{tabular}{|c|c|c|c|c|c|}
\hline & & Age & CRP & ESR & IL-33 \\
\hline CRP & $\begin{array}{l}\text { Rho } \\
\text { P }\end{array}$ & $\begin{array}{c}0.319 \\
0.002 *\end{array}$ & - & - & - \\
\hline ESR & $\begin{array}{l}\text { Rho } \\
\mathrm{P} \\
\end{array}$ & $\begin{array}{c}0.351 \\
0.001 * \\
\end{array}$ & $\begin{array}{c}0.831 \\
0.001 *\end{array}$ & - & - \\
\hline IL-33 & $\begin{array}{l}\text { Rho } \\
p\end{array}$ & $\begin{array}{c}0.295 \\
0.005^{*}\end{array}$ & $\begin{array}{c}0.813 \\
0.001 *\end{array}$ & $\begin{array}{l}0.844 \\
0.001 *\end{array}$ & - \\
\hline $\begin{array}{l}\text { Disease } \\
\text { Duration }\end{array}$ & $\begin{array}{l}\text { Rho } \\
\text { p }\end{array}$ & $\begin{array}{c}0.511 \\
0.001 *\end{array}$ & $\begin{array}{l}0.762 \\
0.001 *\end{array}$ & $\begin{array}{l}0.867 \\
0.001 *\end{array}$ & $\begin{array}{l}0.897 \\
0.001 *\end{array}$ \\
\hline
\end{tabular}

* $P$ value is significant.

IL-33 was recently identified as a member of the IL-1 family and a ligand for the IL-1 family receptor $\mathrm{ST}_{2}{ }^{(2)}$. In patients with RA, immunohistochemistry and in situ hybrid- 
ization have identified IL-33 residing in the synovial cells of inflamed joints ${ }^{(15)}$. IL-33, was detected at high levels in experimental inflammatory arthritis and in the early phases of human RA and was reported to exert profound proinflammatory effects in several experimental autoimmune models. Moreover, administration of IL-33 led to the development of severe inflammatory arthritis, suggesting that IL-33 may be therapeutic target in $\mathrm{RA}^{(16)}$. This cross-sectional study was carried out to measure the level of interleukin 33 (IL-33) in patients with rheumatoid arthritis and assess the correlation of IL-33 level to disease activity, by recruiting 60 patients with RA diagnosed according to the American College of Rheumatology (ACR) 2010 revised criteria. Statistically significant difference was found between RA patients and control. In the present study, IL33 level was also significantly higher in patient's group than the control group ( $\mathrm{p}$.001). Moreover, serum IL-33 level, was significantly higher in patients with active RA (DAS>2.4) than those without activity (DAS $\leq 2.4)$ ( $P$ s.001). Our study met the conclusions of previous studies who reported that serum and synovial fluid (SF) IL-33 levels were higher in severe RA of DAS28>2.4, than in moderate RA with DAS2 8 of $\leq 2.4$ and control groups supporting the idea that IL-33 is implicated in the, pathogenesis of $R A^{(7,17)}$. In the present study, serum IL-33 level positively correlated with disease duration, ESR, CRP and disease severity. The higher levels of IL-33 detected in the sera of RA patients are positively correlated with disease severity, rheumatic factor and the Modified Sharp Score that was used to evaluate bone erosion; the authors therefore suggested that IL-33 may contribute to RA pathogenesis partly through bone erosion ${ }^{(18)}$. However, as IL33 is an inhibitor of bone resorption, it can also be speculated that IL-33 might be se- creted as a result, rather than being the cause, of bone erosion after joint inflammation $^{(19)}$. Despite that, the local expression of IL-33 in the synovium was observed at similar variable levels in RA, PA and $O A$, suggesting that inflamed joints do not represent the only source of elevated serum and SF levels of IL-33 in $R A^{(20)}$. Some recent studies have attempted to evaluate the levels of IL-33 in serum and synovial fluid (SF) in RA patients with the aim of identifying potential biomarkers for the disease. Robust data from different laboratories indicate that the levels of IL-33 in sera and SF samples are significantly increased in RA patients, particularly those with active disease, compared with healthy controls, and osteoarthritis and psoriatic arthritis patients ${ }^{(18)}$. In patients with RA, the serum level of IL-33 and SST2 were significantly higher than that of healthy controls. Consequently, the level of IL-33 in the synovial fluid was significantly higher than that of osteoarthritis patients. All these results confirmed the fact that IL-33/ST2 signaling played a vital role in joint inflammation of human RA and experimental CIA mod$\mathrm{el}^{(21)}$. The role of IL-33 as a pro-inflammatory cytokine in arthritis disease was further confirmed by several reports that exogenous recombinant IL-33 (rIL-33) exacerbated disease severity in $\mathrm{CIA}$ or autoantibody-induced arthritis (AIA) mice, whereas ST2-deficient mice developed attenuated $\mathrm{CIA}$ or AIA together with marked reduction of pro-inflammatory cytokine production (IL-17, TNF- $\alpha$ and IFN$\gamma)^{(22)}$. The present data proved the pleiotropic effect of the new cytokine IL-33 and give new proof on the corroboration of IL-33 in abnormal humoral immunity (anti-inflammatory effect) with insightful proinflammatory outcome despite positive correlation with RF in many searches including us and may ultimately help in understanding the complex issue of auto- 
immunity in RA. Accordingly, counterbalancing of this cytokine may be as a new therapeutic attempt for RA. CRP and ESR, the acute-phase reactants, were used as markers of inflammation, easy to perform, available and the uttermost widely used biological markers for appraising illness activity in $\mathrm{RA}^{(23)}$. The results of the present study showed CRP, ESR, IL-33 were significantly higher in severe RA patients compared to moderate RA patients. Similarly, RA patients who responded to treatment with a TNF inhibitor also showed a reduction in serum IL-33. These important observations suggest that the measurement of IL-33 levels in serum could be a useful marker of RA severity and for monitoring the effect of RA treatment ${ }^{(7)}$. RF is a very old serological marker for diagnosis of RA, taken as a non-specific marker of RA (24). Several studies confirmed that, RF was significantly elevated in RA patients than nonRA patients ${ }^{(25)}$. Also, RF was significantly increased in the serum and synovial fluid of patients with RA in comparison with another inflammatory joint effusion condition as Psoriatic Arthritis and a noninflammatory synovial fluid condition as osteoarthritis $(\mathrm{OA})^{(26)}$. In our study we had few limitations. One limitation of our study is that we did not assess the RF level to limit the cost of the study; another limitation was the insufficient sample size in our study. Taken together, the above data suggest that the IL-33 is critical in promoting arthritogenic inflammation and is a novel therapeutic target for RA disease. However, any treatment involving this pathway will need to be closely monitored as IL-33 also protects bone through inhibiting osteoclast differentiation as shown in in vitro culture ${ }^{(11)}$. In addition, osteoclastogenesis was confirmed to be inhibited in transgenic mice overexpressing IL-33 $33^{(27)}$.

\section{Conclusions}

This study confirmed the importance of IL-33 as a novel marker in assessing the severity and disease activity in RA patients. IL-33 levels were elevated in sera of patients with RA and correlated with disease activity. IL-33 might play an important role in joint inflammation of human RA. We suggest that IL33 could be used as a marker to diagnose and assess the severity of rheumatoid arthritis.

\section{References}

1. Mclnnes IB, and Schett G. (2007): Cytokines in the pathogenesis of rheumatoid arthritis. Nature reviews. Immunology. 7, 429-442.

2. Schmitz J, Owyang A, Oldham E, et al. (2005): IL-33, an interleukin-1-like cytokine that signals via the IL-1 receptor-related protein $\mathrm{ST} 2$ and induces $\mathrm{T}$ helper type 2-associated cytokines. Immunity. 23, 479-490.

3. Duan L, Chen J, Gong F, and Shi G. (2013): The Role of IL-33 in Rheumatic Diseases. Clin Dev Immunol., 2013, 924363, 1-5.

4. Leung BP, Xu D, Culshaw S, Mclnnes IB, and Liew FY. (2004): A novel therapy of murine collagen-induced arthritis with soluble T1/ST2. J Immunol. 173. 145-150.

5. Palmer G, Talabot-Ayer D, Lamacchia, C, et al. (2009): Inhibition of interleukin-33 signaling attenuates the severity of experimental arthritis. Arthritis Rheum, 60, 738-749.

6. Nile CJ, Barksby E, Jitprasertwong $P$, Preshaw PM. and Taylor JJ. (2010): Expression and regulation of interleukin-33 in human monocytes. Immunology. 130, 172-180.

7. Matsuyama $\mathrm{Y}$, Okazaki $\mathrm{H}$, Tamemoto $\mathrm{H}$, et al. (2010): Increased levels of interleukin 33 in sera and synovial fluid from patients with active rheumatoid arthritis. J. Rheumatol., 37, 18-25.

8. Kuroiwa K, Arai T, Okazaki H, Minota S, and Tominaga S. (2001): Identifica- 
tion of human ST2 protein in the sera of patients with autoimmune diseases. Biochem. Biophys. Res. Commun., 284, 1104-1108.

9. O'Dwyer DN, Armstrong ME, Cooke G, Dodd JD, Veale DJ, and Donnelly SC. (2013): Rheumatoid Arthritis associated interstitial lung disease. Eur. J. Intern. Med., 24, 597-603.

10. Parthasarathy V, Kilimozhi D, and Upendar K. (2010): Arthritis - A Review of Clinical Features, Differential Diagnosis. Int J Pharmacy \& Technology. 2, 140.

11. Majithia V, and Geraci SA. (2007): Rheumatoid arthritis: diagnosis and management. Am. J. Med., 120, 936-9.

12. John, S., and Worthington, J. 2001. Genetic epidemiology. Approaches to the genetic analysis of rheumatoid arthritis. Arthritis Res., 3, 216-20.

13. Moon, S.J., Park, J.S., Heo, Y.J., et al. (2013): In vivo action of IL-27: reciprocal regulation of Th17 and Treg cells in collagen-induced arthritis. Exp. Mol. Med., 45: e46.

14. Brennan, F.M., and McInnes, B., (2008): Evidence that cytokines play a role in rheumatoid arthritis. J Clin Invest., 11, 3537-45.

15. Carriere V, Roussel L, Ortega N, et al. (2007): IL-33, the IL-1-like cytokine ligand for ST2 receptor, is a chromatinassociated nuclear factor in vivo. Proc Natl Acad Sci USA. 104, 282-287.

16. Yuan, F.L., Li, X., Lu, W.G., Li, C.W., Xu, R.S. and Dong, J. (2011): IL-33: a promising therapeutic target for RA? Opin. Ther. Targets. 15. 529-34.

17. Tang, S., Huang, H., Hu, F., et al. (2013): Increased IL-33 in Synovial Fluid and Paired Serum Is Associated with Disease Activity and Autoantibodies in RA. Clinical and Developmental Immunology, 2013, 985301.

18. Xiangyang Z, Lutian $Y$, Lin Z, Liping $X$, Hui S, and Jing L. (2012): Increased levels of interleukin-33 associated with bone erosion and interstitial lung diseases in patients with rheumatoid arthritis. Cytokine, 58, 6-9.
19. Schulze J, Bickert T, Beil FT, et al. (2011): Interleukin-33 is expressed in differentiated osteoblasts and blocks osteoclast formation from bone marrow precursor cells. J. Bone Miner. Res., 26, 704-17.

20. Talabot-Ayer, D., McKee, T., Gindre, P., et al. (2013): Distinct serum and synovial fluid interleukin (IL)-33 levels in rheumatoid arthritis, psoriatic arthritis and osteoarthritis. Joint Bone Spine. 79, 32- 37.

21. Hong, Y.S., Moon, S.J., Joo, Y.B., et al. (2011): Measurement of IL-33 and IL-33 receptors ( $\mathrm{SST} 2$ and $\mathrm{ST}_{2} \mathrm{~L}$ ) in patients with rheumatoid arthritis. The Korean Acad Med Sci. 26, 1132-1139.

22. Xu N, Wang Y, Li D, et al. (2010): MDM4 overexpression contributes to synoviocyte proliferation in patients with rheumatoid arthritis. Biochem Biophys Res Commun. 2010 Oct 22; 401 (3):417-21.

23. Nam, J., Villeneuve, E., and Emery, P. (2009): The role of biomarkers in the management of patients with rheumatoid arthritis. Current Rheumatology Reports. 11, 371-377.

24. Singh, U., Vishwanath, A, Verma, PK. et al. (2010): Is RF still a superior test for the diagnosis of RA? Rheumatol. Int., 30, 1115-1119.

25. Li, H, Song, W, Li, Y., et al. (2010): Diagnostic value of anti-CCP antibodies in northern Chinese Han patients with RA and its correlation with disease activity. Clin. Rheumatol., 29, 413-417.

26. Ali OS, Khalifa AIM, and Atia HA. (2014): Levels of Interleukin 33 and Interleukin 27 in Rheumatoid Arthritis and Osteoarthritis Egyptian patients. Int J Advanced Biotechnol and Res. 5, 465-473.

27. Keller J, Catala-Lehnen P, Wintges K, et al. (2012): Transgenic over-expression of interleukin-33 in osteoblasts results in decreased osteoclastogenesis. Biochem. Biophys. Res. Commun., 417, 217-22. 